\title{
Management of the Kidney Transplant Patient with Chronic Hepatitis C Infection
}

\author{
Ignatius Y. S. Tang, ${ }^{1}$ Natasha Walzer, ${ }^{2}$ Nidhi Aggarwal, ${ }^{1}$ \\ Ivo Tzvetanov, ${ }^{3}$ Scott Cotler, ${ }^{2}$ and Enrico Benedetti ${ }^{3}$ \\ ${ }^{1}$ Section of Nephrology, Department of Medicine, University of Illinois at Chicago, Chicago, IL 60612, USA \\ ${ }^{2}$ Section of Hepatology, Department of Medicine, University of Illinois at Chicago, Chicago, IL 60612, USA \\ ${ }^{3}$ Division of Transplant Surgery, Department of Surgery, University of Illinois at Chicago, Chicago, IL 60612, USA
}

Correspondence should be addressed to Ignatius Y. S. Tang, iystang@uic.edu

Received 2 December 2010; Accepted 25 January 2011

Academic Editor: James E. Springate

Copyright ( 2011 Ignatius Y. S. Tang et al. This is an open access article distributed under the Creative Commons Attribution License, which permits unrestricted use, distribution, and reproduction in any medium, provided the original work is properly cited.

Chronic Hepatitis C (HCV) infection is an important cause of morbidity and mortality in patients with end-stage renal disease. Renal transplantation confers a survival advantage in HCV-infected patients. Renal transplant candidates with serologic evidence of HCV infection should undergo a liver biopsy to assess for fibrosis and cirrhosis. Patients with Metavir fibrosis score $\leq 3$ and compensated cirrhosis should be evaluated for interferon-based therapy. Achievement of sustained virological response (SVR) may reduce the risks for both posttransplantation hepatic and extrahepatic complications such as de novo or recurrent glomerulonephritis associated with HCV. Patients who cannot achieve SVR and have no live kidney donor may be considered for HCV-positive kidneys. Interferon should be avoided after kidney transplant except for treatment of life-threatening liver injury, such as fibrosing cholestatic hepatitis. Early detection, prevention, and treatment of complications due to chronic HCV infection may improve the outcomes of kidney transplant recipients with chronic HCV infection.

\section{Introduction}

Hepatitis C (HCV) infection remains highly prevalent in patients with end-stage renal disease (ESRD). The prevalence of HCV in dialysis patients varies among different parts of the world with up to $80 \%$ in developing countries [1] and $1.8 \%$ [2] and $40 \%$ in developed countries [1]. Universal precautions at hemodialysis centers as well as the introduction of screening of blood donors have led to a noticeable decrease in HCV infection in this population; however, nosocomial spread of HCV within dialysis units does continue to occur $[3,4]$. In the United States, the rate of $\mathrm{HCV}$ infection in hemodialysis patients has declined from $10.4 \%$ in 1995 to $7.8 \%$ in 2002 [5], compared to the $1.8 \%$ prevalence observed in the general population [6].

Renal transplantation confers significant survival advantage in HCV-infected patients with end-stage renal disease $[1,2]$. However, HCV-positive kidney transplant recipients experienced lower long-term graft and patient survival compared to their HCV-negative counterparts [7-9]. A meta-analysis of 8 observational studies of 6365 kidney transplant recipients showed that patients with positive $\mathrm{HCV}$ antibodies had a higher rate of death and graft failure after kidney transplantation (relative risk 1.79 and 1.56, resp.) [10]. Hepatocellular carcinoma and liver cirrhosis were the more frequent causes of death in HCV-positive patients [10]. Indeed, liver failure has been reported as a cause of death in $8 \%$ to $28 \%$ of long-term kidney transplant survivors [11-13].

$\mathrm{HCV}$ is also associated with extrahepatic complications: de novo or recurrent glomerulopathy [9], cryoglobulinemic vasculitis, chronic allograft nephropathy [14], posttransplant diabetes mellitus, and sepsis, all of which account for the reduced graft and patient survival [1]. Analysis of the renal transplant cohort of the Australian and New Zealand Dialysis and Transplant Registry in which 140 of 7572 patients (1.8\%) were HCV-positive showed decreased patient survival in the HCV-positive versus HCV-negative kidney transplant recipients: $77 \%$ versus $90 \%$ and $50 \%$ versus $79 \%$ 
at 5 and 10 years, respectively [2]. The higher rate of death in HCV-positive patients was due to cardiovascular disease (hazard ratio (HR) 2.74), malignancy (HR 2.52), and hepatic failure (HR 22.1). HCV-positive patients also had a higher risk of graft loss, the most frequent causes of which were glomerulonephritis, chronic renal allograft nephropathy, and death [2].

The management of kidney transplant recipients with chronic HCV infection is complex. In this paper, we will discuss the following:

(1) the evaluation of HCV in renal transplant candidates and the available treatment options of HCV before transplantation,

(2) the use of HCV-positive donor kidneys,

(3) the monitoring of liver disease progression after transplantation,

(4) the management of HCV-associated extrahepatic complications.

\section{Evaluation of HCV-Positive Renal Transplant Candidates}

All patients undergoing a renal transplant evaluation should be screened for chronic HCV infection with a third generation anti-HCV enzyme-linked immunoassay. If this is positive, confirmation of active infection with a highly sensitive quantitative assay for HCV RNA should be performed. The rate of false negative results is quite low with a third generation immunoassay in patients on hemodialysis [15]. The risk of reactivation in patients who are $\mathrm{HCV}$ antibody positive but HCV RNA negative is extremely rare, even in situations of considerable immunosuppression. A recent study confirmed that patients with previous $\mathrm{HCV}$ infection demonstrated by the presence of HCV antibody, but persistently negative HCV RNA, continued to have no evidence of hepatitis $C$ viral replication in liver biopsies and peripheral blood mononuclear cells up to 16 years after kidney transplantation, despite the use of chronic immunosuppression and often aggressive induction immunosuppression, including antithymocyte globulin and IL-2 receptor blockers [16].

It is generally accepted that HCV-positive patients being evaluated for kidney transplantation should undergo a liver biopsy to assess for the presence of advanced fibrosis, unless there is clear radiological or clinical evidence of portal hypertension or cirrhosis. Cirrhosis has a prevalence of approximately $10 \%$ in ESRD patients with chronic HCV infection [17]. The presence of cirrhosis is usually considered to be a contraindication to renal transplantation, but these patients can be considered for combined liver-kidney transplantation, if there is evidence of decompensated liver disease and portal hypertension. Patients with unremarkable histology can undergo kidney transplantation alone. Patient with Metavir fibrosis score $\leq 3$ and compensated cirrhosis should be considered for interferon- (IFN-) based treatment $[18,19]$, taking into consideration the HCV genotype, side effect profiles of the therapy, and the patient's willingness to comply with the regimens. Liver function tests correlate poorly with histological severity in $\mathrm{HCV}$-positive patients receiving hemodialysis $[20,21]$. Furthermore, patients with ESRD have been found to have less histological activity on liver biopsy, characterized by less inflammation and less fibrosis compared with controls [21, 22].

\section{Liver Biopsy in Hemodialysis Patients}

Percutaneous live biopsy is a safe procedure when performed by experienced operators; however, patients with cirrhosis and other bleeding diatheses can often have an increased risk of hemorrhage requiring hospitalization. A retrospective analysis compared the safety of percutaneous liver biopsy in chronic HCV patients with and without ESRD [22]. Only 1 patient $(1.3 \%)$ with ESRD developed a moderate complication, compared with a $2.1 \%$ complication rate in the control group, with 3 of them having a severe complication. In hemodialysis patients with a suitable coagulation profile (INR $<1.5$, Platelet count $>70,000$, and absence of blood thinners, including aspirin and ibuprofen products), there are currently no data to suggest that percutaneous liver biopsy should be contraindicated. However, patients with ESRD often have coagulation defects and are thought to have an overall increased risk of procedure-related bleeding $[23,24]$.

Transjugular liver biopsy (TJLB) is an alternate means of obtaining liver tissue in patients with coagulopathy and suspected bleeding risk. The results of 46 hemodialysis patients with chronic liver disease undergoing TJLB were compared to 32 hemodialysis patients who had previously had a percutaneous liver biopsy at the same institution [25]. Both techniques yielded adequate specimens for histological diagnosis in all patients. No major complications were observed in the patients who underwent TJLB compared with a $12 \%$ bleeding complication rate in the percutaneous liver biopsy group. Both the INR and platelet count were well above the appropriate threshold for the procedure in both groups. Although this is a much higher complication rate than that seen in previous studies, it certainly raises the question about whether or not TJLB should be the method of choice for obtaining liver tissue in patients on hemodialysis. The TJLB does have the additional benefit of obtaining portal pressure measurements, which may be useful clinically. Moreover, the transjugular approach should be used in patients on peritoneal dialysis.

\section{Noninvasive Markers of Fibrosis}

Non-invasive markers of fibrosis provide a safe method of determining the presence of advanced liver disease, although predictive values vary and many of the assays have not been validated in ESRD patients. The FibroTest (BioPredictive, France), which consists of $\alpha 2$-macroglobulin, haptoglobin, $\gamma$-glutamyl transpeptidase, total bilirubin, and apolipoprotein A1 levels, has been evaluated in both hemodialysis patients and kidney transplant recipients with HCV infection. In 50 ESRD patients, it was found to have a positive predictive value (PPV) of 75\% to detect Metavir F2-F4 for 
scores greater than 0.60 , with a negative predictive value (NPV) of $71 \%$ for scores lower than 0.20 [26]. Recent data yielded contradictory results: the authors suggested that the variability of the components of the FibroTest in hemodialysis patients may explain the difference [27]. For example, lipoprotein metabolism is altered in uremic patients. The literature suggests that a component of uremic serum inhibits hepatic apolipoprotein A-1 synthesis [28]. $\alpha 2$-macroglobulin is an acute-phase reactant and in addition to its association with liver fibrosis, can also be produced at sites of inflammation, which can be induced during the dialysis procedure [29]. The FibroTest is not currently recommended as an alternative to liver biopsy in renal transplant candidates.

An aspartate transaminase (AST) to platelet ratio index (APRI) $<0.40$ accurately identified patients on hemodialysis with Ishak Fibrosis stage 0 or 1 in $93 \%$ of the cases (NPV = 93\%), and all subjects who were misclassified had a fibrosis score of F2. An APRI cutoff $>0.95$ was able to confirm significant fibrosis with a PPV of $66 \%$. If biopsy indication was restricted to APRI scores in the intermediate range ( $>0.40$ and $<0.95$ ), $52 \%$ of liver biopsies could have been correctly avoided [30]. These results were confirmed in a recent prospective analysis that found the APRI to be an accurate and reproducible method of measuring significant fibrosis in hemodialysis patients with chronic HCV [31]. This method of measuring fibrosis needs to be fully validated but could potentially be useful in limiting the number of liver biopsies performed in those being evaluated for kidney transplantation.

\section{HCV Treatment in Renal Transplant Candidates}

Chronic HCV infection leads to significant long-term morbidity and mortality in kidney transplant recipients. The treatment of HCV with interferon (IFN) after transplantation should be avoided because of an increased risk of rejection $[1,3,18]$.

Preemptive HCV treatment of kidney transplant candidates has been shown to improve patient and graft survival [32-34]. Achievement of sustained virological response (SVR) with treatment of HCV not only can prevent progression of liver disease but also reduce the development of posttransplant complications, including $\mathrm{HCV}$-associated nephropathy [35] and new onset diabetes mellitus [36]. SVR is defined as the absence of blood HCV RNA six months after antiviral treatment. Several studies have confirmed that patients who achieve an SVR pretransplant while on hemodialysis with either standard IFN or pegylated-IFN (PEG-IFN) do not experience reactivation of the virus after kidney transplantation, despite high doses of immunosuppression $[37,38]$. Cruzado et al. reported that in $15 \mathrm{HCV}+$ kidney transplant recipients, $10(67 \%)$ of who received IFN pre-transplant, only 1 patient who did not receive treatment and was viremic at the time of transplantation developed de novo glomerulonephritis [35]. In 63 patients who did not receive IFN, $28.7 \%$ had negative HCV-PCR. Twelve of these 63 patients developed de novo glomerulonephritis.
All 12 patients were viremic at time of transplantation [35]. These data provide the impetus to treat HCV-infected individuals while awaiting kidney transplantation.

5.1. Interferon and Ribavirin. Combined PEG-IFN and ribavirin is considered the standard treatment for $\mathrm{HCV}$ infection in patients with normal renal function. Ribavirin is primarily eliminated by the kidney. In patients with glomerular filtration rate less than $30 \mathrm{~mL} / \mathrm{min}$, the area under the blood concentration-time curve is three times higher than that in patients with normal renal function [39]. The main significant adverse effect is hemolytic anemia [39]. Although advanced kidney disease has generally been considered a contraindication to ribavirin use, some clinicians prescribe ribavirin in significantly reduced doses to hemodialysis patients (e.g., $200 \mathrm{mg}$ three times weekly) $[39,40]$. If this approach is taken, patients should be closely monitored for anemia and other adverse events. High doses of recombinant erythropoietin are usually needed to alleviate the anemia $[39,40]$.

A recent meta-analysis of chronic HCV patients on hemodialysis treated with IFN reported a treatment discontinuation rate of $26 \%$ due to adverse events, significantly higher than that reported for IFN-treated nonhemodialysis patients ( $95 \%$ confidence interval (CI), 9 to 14) [41]. This was accompanied, however, by a $41 \%$ SVR rate, much greater than the 10 to $20 \%$ SVR rates observed in previously published studies of nonhemodialysis patients treated with IFN monotherapy $[42,43]$. This is thought to be due to an increased half-life of IFN in hemodialysis patients, resulting in greater plasma IFN levels, increased efficacy and adverse events.

PEG-IFN also has been studied in patients with ESRD. SVR rates between $45 \%$ and $75 \%$ have been observed with PEG-IFN monotherapy in hemodialysis patients [44-46]. Treatment discontinuation rates due to adverse events range from 0 to $33 \%$, with anemia being the most common side effect. It is difficult to draw conclusions on recommendations as the results are quite varied. Treatment efficacy and tolerability of PEG-IFN in patients with ESRD need to be confirmed in a larger prospective clinical trial. Alavian and Tabatabaei performed a meta-analysis of 21 studies on IFN (491 patients) and 12 studies on PEG-IFN (279 patients) to assess the effectiveness of IFN compared to PEG-IFN monotherapy [47]. The pooled SVR for IFN and PEG-IFN was $39.1 \%$ (95\% CI, 32.1 to 46.1 ) and $39.3 \%$ (95\% CI, 26.5 to 52.1$)$, respectively. The pooled dropout rates were $22.6 \%$ (95\% CI, 10.4 to 34.8 ) and $29.7 \%$ (95\% CI, 21.7 to 37.7), respectively [47]. Hence, whether PEG-IFN confers additional benefits in terms of SVR and adverse events is unclear.

There are limited studies of the use of combined IFN or PEG-IFN with ribavirin in patients with advanced kidney disease. Fabrizi et al. conducted a meta-analysis of 10 clinical trials (only one of which was a controlled study) involving 151 patients, $97.4 \%$ of who were hemodialysis patients [48]. The summary estimates for SVR and dropout rate were $56 \%$ (95\% CI, 28 to 84 ) and $25 \%$ (95\% CI, 10 to 40 ), respectively. The most frequent adverse events 
were anemia (26\%) and heart failure (9\%) [48]. However, there was significant heterogeneity in SVR and drop-out rates among the studies. Prospective controlled trials are needed to assess the efficacy and tolerability of combined IFN or PEG-IFN with ribavirin compared to IFN or PEG-IFN monotherapy.

It is well known that HCV genotype can significantly impact the response rate to IFN and ribavirin. Hence, it is routine to obtain genotype testing prior to initiation of treatment. Studies have shown that the prevalence of $\mathrm{HCV}$ genotypes in patients with ESRD is quite similar to that in the general population, with the majority of patients being infected with either genotype 1a or 1b [49]. In addition, the HCV genotype does not seem to have an impact on survival in kidney transplant recipients [49]. There are virtually no data on comparing treatment response of the various genotypes in patients on hemodialysis, but small studies do suggest that genotype 1 patients are able to achieve an SVR anywhere from $28 \%$ to $75 \%$ of the time with a 48-week course of PEG-IFN monotherapy [50-52].

For HCV-infected patients with chronic kidney disease on maintenance hemodialysis, the 2008 KDIGO guidelines recommend monotherapy with standard interferon that is dose adjusted for a glomerular filtration rate less than $15 \mathrm{~mL} / \mathrm{min}$ [19]. Strong data are not available to make dosing and treatment duration recommendations. However, a regimen of three million units of IFN $\alpha$-2b given subcutaneously three times per week or PEG-IFN $\alpha$-2a $135 \mathrm{mcg}$ weekly for 6 to 12 months appears to be safe and effective in inducing an SVR [39]. Ribavirin can be initiated at $200 \mathrm{mg}$ daily or $200 \mathrm{mg}$ three times weekly with close monitoring of anemia and adverse events in patients who have no virologic response to IFN or PEG-IFN [39, 40, 48].

5.2. Protease Inhibitors. The incomplete effectiveness of treatment with PEG-IFN and ribavirin has prompted the development of selective inhibitors of HCV. The NS3/4A protease inhibitors, which specifically target viral replication, have been shown to have potent antiviral activity in HCV replicon assays. In initial clinical studies, the NS3/4A protease inhibitors have been well tolerated with substantial antiviral activity [53]. Results of two phase II studies-PROVE 1 and PROVE 2-have shown safety and efficacy of the triple regimen of IFN $\alpha-2 \mathrm{a}$, ribavirin, and telaprevir in patients with untreated chronic HCV (genotype 1) [54, 55]. PROVE2 also included a telaprevir and IFN $\alpha$ (without ribavirin) arm that was treated for 12 weeks. Patients who received telaprevir and PEG-IFN without ribavirin were less likely to achieve HCV RNA suppression and more likely to relapse than were those who received the triple combination.

There are no data on the safety and efficacy of the HCV protease inhibitors, telaprevir and boceprevir, in patients with ESRD. The HIV protease inhibitors, however, are hepatically cleared, and appear to be safe in patients with chronic renal failure, as well as for those on hemodialysis $[56,57]$. Additional pharmacokinetic studies need to be done in order to define dosing recommendations in patients on hemodialysis.

\section{Use of HCV Antibody-Positive Donor Kidneys}

While it is well accepted that kidneys from HCV-positive donors should not be transplanted into HCV-negative recipients, the use of kidneys from $\mathrm{HCV}$-infected donors for transplant in $\mathrm{HCV}$-positive recipients has been controversial $[1,18,19]$. The use of HCV-positive kidneys may shorten the waiting times for ESRD patients with chronic HCV infection and may increase the availability of $\mathrm{HCV}$-negative kidneys to HCV-negative recipients. Several single center studies had shown similar short-term graft and patient survival with no increase in acute rejection compared to those who received HCV-negative kidneys [58].

A recent analysis of the UNOS database from 1995 to 2009 showed that HCV-positive kidneys were 2.6 times more likely to be discarded [59]. Twenty-nine percent of 6830 patients received HCV-positive kidneys. On average, the waiting time decreased from 856 to 469 days. HCVpositive kidney recipients had a 1.29 times risk of death $(95 \%$ CI $1.15-1.45, P<.001$ ) [59]. However, this hazard ratio only translated into a difference of $1 \%$ in 1-year survival between $\mathrm{HCV}$-negative and $\mathrm{HCV}$-positive kidney recipients (94\% versus $93 \%$ ) and a $2 \%$ difference in 3 year survival ( $85 \%$ versus $83 \%$ ). Interestingly, non-African Americans had a higher death risk when receiving an $\mathrm{HCV}$-positive kidney (hazard ratio 1.6, 95\% CI 1.35-1.90, $P<.01$ ) [59]. African Americans, patients older than 60, diabetics and highly sensitized patients did not have significantly increased risk of death. Similarly, recipients of kidneys from HCV-positive donors had a 1.18 times risk of graft loss, compared to those receiving HCV-negative kidneys [59]. Patients younger than 60 , those without diabetes, those with panel reactive antibodies $<80 \%$, and those with BMI $<35 \mathrm{~kg} / \mathrm{m}^{2}$ were associated with an increased hazard of graft loss.

The early experience in 2 transplant centers in Spain showed transmission of HCV RNA in HCV antibodypositive recipients who were negative for HCV RNA [60]. The policy was then changed to only transplant HCVpositive kidneys to $\mathrm{HCV}$-positive recipients with viremia. An update of the long-term experience from Spain using HCV-positive kidney donor was reported [60]. There was no difference in the 5- and 10-year patient survival between 162 recipients of $\mathrm{HCV}$-positive kidneys versus 306 recipients of HCV-negative kidneys: $84.8 \%$ and $72.7 \%$ versus $86.6 \%$ and $76.5 \%$, respectively, $P=.250$ [60]. Three deaths in HCV-positive kidney recipients and 2 in HCV-negative kidney recipients were related to liver disease. There was no statistically significant difference in the 5- and 10-year deathcensored graft survival, $69 \%$ and $47 \%$ in the HCV-positive kidney recipients versus $72.7 \%$ and $58.5 \%$ in the HCVnegative kidney recipients, $P=.05$ [60]. The incidence of decompensated liver disease was also not different between the 2 groups: $10.3 \%$ versus $6.2 \%$. Donor HCV serology was not found to be a risk factor for death, graft failure and severe liver disease [60]. Hence, transplantation of kidney from an $\mathrm{HCV}$-positive donor to an $\mathrm{HCV}$-positive recipient with detectable HCV RNA appeared to be safe with satisfactory outcome [19]. Nucleic acid test should be done in HCVpositive kidney donors if available. 
The effect of superinfection with a different HCV geno-type when an HCV-positive kidney is transplanted into an HCV-positive recipient remains to be studied [18]. Severe HCV infection with genotype 1 has been reported in a 3-time kidney transplant recipient who was chronically infected with HCV genotype 2a [61]. Hence, the benefits and risks of receiving $\mathrm{HCV}$-positive donor kidneys should be discussed and explained with the potential renal transplant candidate so that a well informed decision can be made [19]. Of course, the option of living donor kidney transplantation should always be encouraged.

\section{HCV and Immunosuppression}

The impact of chronic immunosuppression on the course of HCV infection in renal transplant recipients is not well defined. Corticosteroids at high doses have been shown to increase HCV viremia, probably by upregulation of cell entry factors such as occludin and scavenger receptor class B type 1 , and result in HCV dissemination [62]. The effect of low maintenance doses is unclear.

The safety and efficacy of Campath-1 induction and a steroid-free maintenance regimen with tacrolimus and mycophenolate were evaluated in $24 \mathrm{HCV}$-positive kidney transplant recipients [63]. During a mean followup of 15 months, there were 3 graft loss and 3 deaths (unrelated to liver disease), with $79 \%$ allograft survival and $83 \%$ patient survival [63]. Abnormal liver function tests were found in 3 patients, one due to fatty liver, one had been treated with steroids for acute rejection, and one treated with leflunomide for BK virus. No exacerbation of HCV infection was reported [63].

The efficacy and safety of a rapid steroid tapering regimen under antilymphocyte antibody induction was studied in HCV-positive kidney transplant recipients [64]. Twelve patients received induction treatment with antilymphocyte antibody followed by rapid steroid withdrawal over 6 days and maintained on calcineurin inhibitor and mycophenolate. Seventeen patients received standard steroid taper. On followup (median followup of 12 months in the rapid steroid taper group and 21 months in the historical control group), there was no difference in patient survival $92 \%$ versus $92 \%$ and graft survival $92 \%$ versus $82 \%$ between the rapid steroid withdrawal and the standard steroid taper groups [64]. There was also no difference in acute rejection episodes and post-transplant liver abnormalities between the 2 groups.

Cyclosporine has been shown to have anti-HCV activity in cultured cells [65]. However, its effect on HCV clinically is unclear. Whether mycophenolate is detrimental in terms of graft and patient survival in kidney transplant patients with chronic HCV infection is unclear [19]. In a recent registry data analysis, the use of mycophenolate as part of maintenance immunosuppression was associated with better patient survival [66]. This beneficial effect was not demonstrated with cyclosporine, tacrolimus, sirolimus, azathioprine and steroids. Interestingly, in the same study, the use of antilymphocyte antibody induction was not associated with lower patient survival [66]. In a small study of $7 \mathrm{HCV}$-positive kidney transplant recipients treated with rituximab for de novo cryoglobulinemic glomerulonephritis, there were no significant changes in aminotransferase, bilirubin, and viral load 6 months post-rituximab therapy [67].

Though most immunosuppressive agents increase viral replication, the implication is unclear. Selection of specific immunosuppressive regimens should be individualized and balanced between the potential effect on $\mathrm{HCV}$-associated hepatic and extra-hepatic complications [19]. Prospective controlled studies are needed to assess the impact of various immunosuppressive regimens on chronic HCV infection in kidney transplant recipients.

\section{Evaluating HCV after Kidney Transplantation}

The natural history of HCV-related liver disease in kidney transplant recipients is controversial; however, there are some data to suggest that progression rate to cirrhosis may be slower in $\mathrm{HCV}$-positive renal transplant recipients compared to $\mathrm{HCV}$-positive controls without kidney disease [68]. One analysis of $51 \mathrm{HCV}$-RNA-positive kidney transplant patients who underwent a mean of three consecutive liver biopsies after transplantation every 3-4 years found that liver fibrosis progressed at a rate of $0.09 \pm 0.03$ Metavir units/year [69]. Only 3 of the 51 patients developed cirrhosis during long-term followup. Nonetheless, liver biopsy remains the gold standard to assess the progression of HCV-related liver disease after kidney transplantation. The risks of percutaneous liver biopsy as previously discussed and the presence of sampling error have led to the evaluation of both the laboratory and radiological methods of assessing liver fibrosis. None of these, however, have been validated in the postkidney transplant population.

Ultrasound elastography, known commercially as Fibroscan, has been evaluated as a noninvasive method to evaluate liver fibrosis in kidney transplant patients. It is a radiologic technique that uses a modified ultrasound probe to measure the velocity of a shear wave created by a vibratory source [70]. Estimates of stiffness of the liver by ultrasound correlate with fibrosis stage [71]. All patients with low Fibroscan scores $(n=13 ; 5.2 \mathrm{kPa})$ exhibited the lowest stage of liver fibrosis by biopsy accompanied by normal liver function. In contrast, patients with high Fibroscan scores $(n=6 ; 11 \mathrm{kPa})$ showed a severe form of liver fibrosis with clinical evidence of portal hypertension [72]. Fibroscan appears to be a promising test in following fibrosis progression in kidney transplant recipients, but it has not yet been approved for use in the United States.

The KDIGO guidelines recommended monthly measurement of liver function test in the first 6 months and then every 3 months thereafter [19]. Patients with worsening trend in liver enzymes should be referred for Hepatology evaluation. In patients with cirrhosis on liver biopsy, annual liver ultrasound and alpha-fetoprotein measurement should be considered [19]. 


\section{HCV and De Novo and Recurrent Glomerular Diseases}

Chronic infection with HCV has been linked to the pathogenesis of glomerular diseases, both in native as well as transplanted kidneys. There is an increased incidence of proteinuria among $\mathrm{HCV}$-positive renal transplant patients compared to HCV-negative patients [73].

9.1. HCV-Associated Glomerular Lesions after Transplantation. Membranoproliferative glomerulonephritis (MPGN), with or without cryoglobulinemia is the most common renal lesion associated with chronic HCV infection in the renal transplant recipients $[74,75]$. The second most important de novo glomerular disease frequently seen in such cases is membranous glomerulonephritis (MGN) [75-77]. The pathogenesis of these lesions is similar to what occurs in native kidneys-deposition of $\mathrm{HCV}$-protein containing immune-complexes in the glomeruli, which occurs despite immunosuppressive therapy. A possible explanation of this phenomenon is that immunosuppression increases $\mathrm{HCV}$ viral load and reduces immunoglobulin synthesis, leading to an imbalance of antigen-antibody complex status, which interferes with their clearance and leads to their deposition in the allograft [78]. Kamar et al. have suggested that HCVpositive patients who developed de novo glomerulonephritis have a higher immune response and/or particular cytokine production rather than a direct effect of $\mathrm{HCV}$ on kidney cells [79]. In addition to MPGN and MGN, Baid et al. described the association of $\mathrm{HCV}$ infection with the development of de novo thrombotic microangiopathy in the renal allograft, especially in patients with pretransplant anticardiolipin antibodies [80]. Cosio et al. also showed a high incidence of acute transplant glomerulopathy in HCV-positive kidney transplants [81].

9.2. Posttransplant Management of HCV-Positive Renal Transplants. As there is increased risk of development of glomerulopathy and graft loss in HCV-positive patients, close monitoring for the development of proteinuria is required. It is recommended that a baseline urinalysis and urine proteinto-creatinine ratio should be obtained within the first two weeks after transplant or once the renal function is stable [19]. Screening for proteinuria should be done every 3-6 months for the first year and every 6 months thereafter. If a patient develops significant proteinuria (defined as urine protein-to-creatinine ratio of $>1.0$ or 24 -hour urine protein $>1.0 \mathrm{~g}$ on two or more occasions) or microscopic hematuria (without any other identified cause), a renal allograft biopsy should be performed [19]. The kidney biopsy should be studied with light microscopy, immunofluorescence techniques and electron microscopy. Differentiation of MPGN from chronic transplant glomerulopathy should be done as this may influence subsequent therapy. Immunological and serologic studies, especially cryoglobulin and complement levels, may be useful in the diagnosis of cryoglobulinemic MPGN. Electron microscopy can help to distinguish these entities, especially in patients with noncryoglobulinemic MPGN. The presence of large subendothelial electron-dense deposits is diagnostic of MPGN, whereas the mere presence of thickening and duplication of glomerular basement membrane favors the diagnosis of chronic transplant glomerulopathy.

Pre-transplant use of IFN-based therapies for treatment of HCV infection may prevent the development of posttransplant glomerulonephritis. In kidney transplant recipients diagnosed with $\mathrm{HCV}$-associated glomerulopathy, use of interferon- $\alpha$-based therapies should be generally avoided as use of IFN in the setting of kidney transplant is associated with an increased risk of renal allograft rejection, including antibody-mediated rejection $[1,19,82]$. Antiviral therapies such as ribavirin can be used in kidney transplant recipients with HCV-associated glomerulopathy [83]. Ribavirin helps to reduce proteinuria, but it does not lead to viral clearance. Other nonspecific antiproteinuric therapies such as blockade of the renin-angiotensin-aldosterone system with angiotensin-converting enzyme inhibitors and angiotensinreceptor blockers may also be used as tolerated. In such cases, careful monitoring of renal function, serum potassium and hemoglobin levels is required.

In cryoglobulinemic glomerulonephritis, high-dose intravenous pulse steroids followed by steroid taper can be used for the treatment of severe nephritic or nephrotic proteinuria associated with renal dysfunction. In addition, cytotoxic drugs like cyclophosphamide have been used for severe cases. Cyclophosphamide improves renal disease by suppressing B-lymphocyte stimulation and cryoglobulin production. However, such therapies are associated with a high rate of morbidity and mortality even in immunocompetent patients [84]. In addition, the efficacy of these treatments remains unclear. In patients with native kidney cryoglobulinemic glomerulonephritis, plasma exchange three times a week for 2 to 3 weeks has been successfully used [85]. Plasma exchange aims to remove circulating cryoglobulins, inflammatory mediators and toxins. However, there are no definitive data for its use in renal transplant patients for this indication. Regardless, plasma exchange is extensively and safely used for other indications in this population.

Rituximab is a chimeric monoclonal antibody that binds to the B cell surface antigen CD 20 and leads to rapid depletion of B-cells in the peripheral blood. Thus, it interferes with monoclonal IgM production, cryoglobulin synthesis and renal deposition of immune complexes. Rituximab has been used successfully for the treatment of cryoglobulinemia in non-immunocompromised patients [86, 87]. Basse et al. used rituximab in 7 renal transplant patients ( 5 of who were HCV positive) with MPGN associated with mixed cryoglobulinemia [88]. Use of 2-4 doses of rituximab was associated with a sustained clearance of cryoglobulins and a significant reduction in proteinuria. However, 2 of these patients developed severe infections. Prospective trials to fully investigate the role of rituximab in this population are warranted.

Novel therapies like imatinib are being studied in animal models for the treatment of cryoglobulinemia and MPGN. Imatinib is a tyrosine kinase inhibitor. In mice with cryoglobulinemia and MPGN, use of imatinib led to the reduction in cryoglobulin production and the reversal of renal and systemic lesions [89]. Further studies in humans, specifically in renal transplant patients, are still required. 
In summary, de novo and recurrent glomerulopathies are common in renal transplant patients with HCV infection and are associated with poorer allograft and patient survival. These patients should be closely monitored for the development of complications from HCV infection. A careful evaluation to identify the cause of renal dysfunction should be undertaken in these patients. Use of interferon therapy should be avoided. All patients should receive antiproteinuric therapy as tolerated and antiviral treatment may be considered. High-dose corticosteroids and plasma exchange may be used in acute and severe cases. Rituximab may be tried in refractory cases.

\section{HCV and New Onset Diabetes after Transplantation}

HCV has been associated with diabetes mellitus in both preand posttransplant patients. The incidence and prevalence of new onset diabetes after transplantation (NODAT) are variable because of the different definitions used, the organ transplanted, and the duration of followup [90]. A metaanalysis of multiple clinical studies reported the incidence of NODAT between $7.9 \%$ and 50\% [36]. Bloom et al. reported a prevalence of $39.4 \%$ in $\mathrm{HCV}$-positive kidney transplant recipients compared to $9.8 \%$ of $\mathrm{HCV}$-negative patients, $P<.0005$ [91]. Kasiske et al. found that NODAT increased the risks of both graft failure (HR 1.63, $P<.0001$ ) and death (HR 1.87, $P<.0001$ ) [92]. The mechanisms for $\mathrm{HCV}$-associated diabetes mellitus are multiple and include increased insulin resistance, impaired insulin sensitivity [93], reduced hepatic glucose uptake and glycogenesis, and direct viral cytopathic effect on pancreatic $\beta$ cells [90]. In transplant patients other mechanisms may come into play: obesity, older age, African American and Hispanic ethnicities, metabolic syndrome, and immunosuppressive therapy, including calcineurin inhibitors [94] and steroids [90]. Bloom et al. reported that $57.8 \%$ of HCV-positive patients treated with tacrolimus developed NODAT compared with $7.7 \%$ on cyclosporine therapy $(P<.0001)$ [91]. Interestingly, there was no difference in NODAT between the HCVnegative recipients using tacrolimus versus cyclosporine $10 \%$ and $9.4 \%$, respectively, $P=.521$ [91].

Early diagnosis and appropriate treatment of NODAT are important. Patients diagnosed with diabetes mellitus as defined by the American Diabetic Association criteria should be referred to the diabetologist for further evaluation and management [19]. Steroid minimization and reduction in tacrolimus or conversion to cyclosporine may help to minimize the risk of NODAT. Increased physical activity, weight loss, and treatment of the various components of the metabolic syndrome are important aspects in the management of NODAT. Achievement of SVR with antiviral treatment can reduce the risk of HCV-associated NODAT.

\section{Conclusion}

Chronic HCV infection is an important cause of morbidity and mortality in patients with ESRD. Kidney transplantation confers a survival advantage in HCV-infected patients.
Renal transplant candidates with serologic evidence of HCV infection should undergo a liver biopsy to assess for fibrosis and cirrhosis. Patients with Metavir fibrosis score $\leq 3$ and compensated cirrhosis should be evaluated for IFNbased therapy. Achievement of SVR may reduce the risks for both the hepatic and extrahepatic complications such as glomerulonephritis and NODAT associated with HCV. Patients who cannot achieve SVR and have no live donor may be considered for HCV-positive kidneys. Interferon should be avoided after kidney transplant except for treatment of life-threatening liver injury, such as fibrosing cholestatic hepatitis. Early detection, prevention, and treatment of complications due to chronic HCV infection can improve the outcomes of HCV-positive kidney transplant recipients.

\section{References}

[1] P. Martin and F. Fabrizi, "hepatitis C virus and kidney disease," Journal of Hepatology, vol. 49, no. 4, pp. 613-624, 2008.

[2] D. R. Scott, J. K. W. Wong, and T. S. Spicer, "Adverse impact of hepatitis $\mathrm{C}$ virus infectin on renal replacement therapy and renal transplant patients in Australia and New Zealand," Tranplantation, vol. 90, no. 11, pp. 1165-1171, 2010.

[3] N. A. Terrault and D. B. Adey, "The kidney transplant recipient with hepatitis C infection: pre- and posttransplantation treatment," Clinical Journal of the American Society of Nephrology, vol. 2, no. 3, pp. 563-575, 2007.

[4] S. M. Alavian, "A shield against a monster: hepatitis C in hemodialysis patients," World Journal of Gastroenterology, vol. 15, no. 6, pp. 641-646, 2009.

[5] L. Finelli, J. T. Miller, J. I. Tokars, M. J. Alter, and M. J. Arduino, "National surveillance of dialysis-associated diseases in the United States, 2002," Seminars in Dialysis, vol. 18, no. 1, pp. 52-61, 2005.

[6] E. J. Okoh, J. R. Bucci, J. F. Simon, and S. A. Harrison, "HCV in patients with end-stage renal disease," American Journal of Gastroenterology, vol. 103, no. 8, pp. 2123-2134, 2008.

[7] D. Serón, M. Arias, J. M. Campistol, and J. M. Morales, "Late renal allograft failure between 1990 and 1998 in Spain: a changing scenario," Transplantation, vol. 76, no. 11, pp. 15881594, 2003.

[8] J. M. Morales, B. Domínguez-Gil, D. Sanz-Guajardo, J. Fernández, and F. Escuin, "The influence of hepatitis B and hepatitis $\mathrm{C}$ virus infection in the recipient on late renal allograft failure," Nephrology Dialysis Transplantation, vol. 19, supplement 3, pp. iii72-iii76, 2004.

[9] J. M. Cruzado, M. Carrera, J. Torras, and J. M. Grinyó, "hepatitis $\mathrm{C}$ virus infection and de novo glomerular lesions in renal allografts," American Journal of Transplantation, vol. 1, no. 2, pp. 171-178, 2001.

[10] F. Fabrizi, P. Martin, V. Dixit, S. Bunnapradist, and G. Dulai, "hepatitis $\mathrm{C}$ virus antibody status and survival after renal transplantation: meta-analysis of observational studies," American Journal of Transplantation, vol. 5, no. 6, pp. 14521461, 2005.

[11] J. A. Fishman, R. H. Rubin, M. J. Koziel, and B. J. G. Periera, "hepatitis C virus and organ transplantation," Transplantation, vol. 62, no. 2, pp. 147-154, 1996.

[12] M. R. Weir, R. L. Kirkman, T. B. Strom, and N. L. Tilney, "Liver disease in recipients of long-functioning renal allografts," Kidney International, vol. 28, no. 5, pp. 839-844, 1985. 
[13] Y. Kokado, S. Takahara, N. Ichimaru et al., "Clinical outcome of HCV infection after renal transplantation," Transplantation Proceedings, vol. 32, no. 7, pp. 1940-1943, 2000.

[14] I. M. Mahmoud, A. F. Elhabashi, E. Elsawy, A. A. El-Husseini, G. E. Sheha, and M. A. Sobh, "The impact of hepatitis C virus viremia on renal graft and patient survival: a 9-year prospective study," American Journal of Kidney Diseases, vol. 43, no. 1, pp. 131-139, 2004.

[15] H. Hinrichsen, G. Leimenstoll, G. Stegen, H. Schrader, U. R. Fölsch, and W. E. Schmidt, "Prevalence and risk factors of hepatitis $\mathrm{C}$ virus infection in haemodialysis patients: a multicentre study in 2796 patients," Gut, vol. 51, no. 3, pp. 429-433, 2002.

[16] F. Nicot, N. Kamar, B. Mariamé, L. Rostaing, C. Pasquier, and J. Izopet, "No evidence of occult hepatitis $\mathrm{C}$ virus (HCV) infection in serum of HCV antibody-positive HCV RNAnegative kidney-transplant patients," Transplant International, vol. 23, no. 6, pp. 594-601, 2010.

[17] P. Martin, D. Carter, F. Fabrizi et al., "Histopathological features of hepatitis C in renal transplant candidates," Transplantation, vol. 69, no. 7, pp. 1479-1484, 2000.

[18] S. Tomé, J. Pascual, J. D. Pirsch, A. Musat, and M. R. Lucey, "hepatitis C infection in kidney transplantation," Transplantation Reviews, vol. 21, no. 2, pp. 86-96, 2007.

[19] "KDIGO clinical practice guidelines for the prevention, diagnosis, evaluation, and treatment of hepatitis $\mathrm{C}$ in chronic kidney disease," Kidney International. Supplement, vol. 109, pp. S1-S99, 2009.

[20] P. Mathurin, C. Mouquet, T. Poynard et al., "Impact of hepatitis B and C virus on kidney transplantation outcome," Hepatology, vol. 29, no. 1, pp. 257-263, 1999.

[21] S. J. Cotler, G. Diaz, S. Gundlapalli et al., "Characteristics of hepatitis C in renal transplant candidates," Journal of Clinical Gastroenterology, vol. 35, no. 2, pp. 191-195, 2002.

[22] S. Pawa, M. Ehrinpreis, M. Mutchnick, J. Janisse, R. Dhar, and F. A. Siddiqui, "Percutaneous liver biopsy is safe in chronic hepatitis C patients with end-stage renal disease," Clinical Gastroenterology and Hepatology, vol. 5, no. 11, pp. 1316-1320, 2007.

[23] A. L. Weigert and A. I. Schafer, "Uremic bleeding: pathogenesis and therapy," American Journal of the Medical Sciences, vol. 316, no. 2, pp. 94-104, 1998.

[24] M. Šabovič, B. Salobir, I. P. Zupan, P. Bratina, V. Bojec, and J. B. Ponikvar, "The influence of the haemodialysis procedure on platelets, coagulation and fibrinolysis," Pathophysiology of Haemostasis and Thrombosis, vol. 34, no. 6, pp. 274-278, 2006.

[25] A. Ahmad, F. Hasan, S. Abdeen et al., "Transjugular liver biopsy in patients with end-stage renal disease," Journal of Vascular and Interventional Radiology, vol. 15, no. 3, pp. 257260, 2004.

[26] A. Varaut, H. Fontaine, J. Serpaggi et al., "Diagnostic accuracy of the fibrotest in hemodialysis and renal transplant patients with chronic hepatitis C virus," Transplantation, vol. 80, no. 11, pp. 1550-1555, 2005.

[27] M. Canbakan, H. Senturk, B. Canbakan et al., "Validation of biochemical markers for the prediction of liver fibrosis and necroinflammatory activity in hemodialysis patients with chronic hepatitis C," Nephron-Clinical Practice, vol. 117, no. 3, pp. c289-c295, 2010.

[28] V. S. Kamanna, M. L. Kashyap, R. Pai et al., "Uremic serum subfraction inhibits apolipoprotein A-I production by a human hepatoma cell line," Journal of the American Society of Nephrology, vol. 5, no. 2, pp. 193-200, 1994.
[29] T. Poynard, F. Imbert-Bismut, V. Ratziu et al., "Biochemical markers of liver fibrosis in patients infected by hepatitis $C$ virus: longitudinal validation in a randomized trial," Journal of Viral Hepatitis, vol. 9, no. 2, pp. 128-133, 2002.

[30] L. L. Schiavon, J. L. N. Schiavon, R. J. Carvalho Filho et al., "Simple blood tests as noninvasive markers of liver fibrosis in hemodialysis patients with chronic hepatitis $\mathrm{C}$ virus infection," Hepatology, vol. 46, no. 2, pp. 307-314, 2007.

[31] C. H. Liu, C. C. Liang, C. J. Liu et al., "The ratio of aminotransferase to platelets is a useful index for predicting hepatic fibrosis in hemodialysis patients with chronic hepatitis C," Kidney International, vol. 78, no. 1, pp. 103-109, 2010.

[32] F. Fabrizi, G. Dulai, V. Dixit, S. Bunnapradist, and P. Martin, "Meta-analysis: interferon for the treatment of chronic hepatitis C in dialysis patients," Alimentary Pharmacology and Therapeutics, vol. 18, no. 11-12, pp. 1071-1081, 2003.

[33] F. Fabrizi, F. F. Poordad, and P. Martin, "hepatitis C infection and the patient with end-stage renal disease," Hepatology, vol. 36, no. 1, pp. 3-10, 2002.

[34] M. W. Russo, R. Ghalib, S. Sigal, and V. Joshi, "Randomized trial of pegylated interferon $\alpha$-2b monotherapy in haemodialysis patients with chronic hepatitis C," Nephrology Dialysis Transplantation, vol. 21, no. 2, pp. 437-443, 2006.

[35] J. M. Cruzado, T. Casanovas-Taltavull, J. Torras, C. Baliellas, S. Gil-Vernet, and J. M. Grinyó, "Pretransplant interferon prevents hepatitis $\mathrm{C}$ virus-associated glomerulonephritis in renal allografts by HCV-RNA clearance," American Journal of Transplantation, vol. 3, no. 3, pp. 357-360, 2003.

[36] F. Fabrizi, P. Martin, V. Dixit, S. Bunnapradist, F. Kanwal, and G. Dulai, "Post-transplant diabetes mellitus and HCV seropositive status after renal transplantation: meta-analysis of clinical studies," American Journal of Transplantation, vol. 5, no. 10, pp. 2433-2440, 2005.

[37] T. Casanovas-Taltavull, C. Baliellas, C. Benasco et al., "Efficacy of interferon for chronic hepatitis $\mathrm{C}$ virus-related hepatitis in kidney transplant candidates on hemodialysis: results after transplantation," American Journal of Gastroenterology, vol. 96, no. 4, pp. 1170-1177, 2001.

[38] N. Kamar, O. Toupance, M. Buchler et al., "Evidence that clearance of hepatitis $\mathrm{C}$ virus RNA after alpha-interferon therapy in dialysis patients is sustained after renal transplantation," Journal of the American Society of Nephrology, vol. 14, pp. 2092-2098, 2003.

[39] N. Perico, D. Cattaneo, B. Bikbov, and G. Remuzzi, "hepatitis $\mathrm{C}$ infection and chronic renal diseases," Clinical Journal of the American Society of Nephrology, vol. 4, no. 1, pp. 207-220, 2009.

[40] H. Weclawiak, N. Kamar, A. Ould-Mohamed et al., "Treatment of chronic hepatitis $\mathrm{C}$ virus infection in dialysis patients: an update," Hepatitis Research and Treatment, vol. 2010, Article ID 267412, 6 pages, 2010.

[41] C. E. Gordon, K. Uhlig, J. Lau, C. H. Schmid, A. S. Levey, and J. B. Wong, "Interferon treatment in hemodialysis patients with chronic hepatitis $\mathrm{C}$ virus infection: a systematic review of the literature and meta-analysis of treatment efficacy and harms," American Journal of Kidney Diseases, vol. 51, no. 2, pp. 263277, 2008.

[42] J. G. Mchutchison, S. C. Gordon, E. R. Schiff et al., "Interferon alfa-2b alone or in combination with ribavirin as initial treatment for chronic hepatitis C," New England Journal of Medicine, vol. 339, no. 21, pp. 1485-1492, 1998.

[43] T. Poynard, P. Marcellin, S. S. Lee et al., "Randomised trial of interferon $\alpha 2 \mathrm{~b}$ plus ribavirin for 48 weeks or for 24 weeks versus interferon $\alpha 2 \mathrm{~b}$ plus placebo for 48 weeks for treatment 
of chronic infection with hepatitis C virus," The Lancet, vol. 352, no. 9138, pp. 1426-1432, 1998.

[44] O. F. Kokoglu, H. Uçmak, S. Hosoglu et al., "Efficacy and tolerability of pegylated-interferon alpha-2a in hemodialysis patients with chronic hepatitis C," Journal of Gastroenterology and Hepatology, vol. 21, no. 3, pp. 575-580, 2006.

[45] T. Werner, B. Aqel, V. Balan et al., "Treatment of hepatitis C in renal transplantation candidates: a single-center experience," Transplantation, vol. 90, pp. 407-411, 2010.

[46] C. H. Liu, C. C. Liang, J. W. Lin et al., "Pegylated interferon $\alpha$ 2a versus standard interferon $\alpha$-2a for treatment-naïve dialysis patients with chronic hepatitis C: a randomised study," Gut, vol. 57, no. 4, pp. 525-530, 2008.

[47] S. M. Alavian and S. V. Tabatabaei, "Meta-analysis of factors associated with sustained viral response in patients on hemodialysis treated with standard or pegylated interferon for hepatitis C infection," Iranian Journal of Kidney Diseases, vol. 4, no. 3, pp. 181-194, 2010.

[48] F. Fabrizi, V. Dixit, P. Martin, and P. Messa, "Combined antiviral therapy of hepatitis $\mathrm{C}$ virus in dialysis patients: metaanalysis of clinical trials," The Journal of Viral Hepatitis. In press.

[49] S. N. Natov, J. Y. Lau, R. Ruthazer et al., "hepatitis C virus genotype does not affect patient survival among renal transplant candidates. The New England Organ Bank hepatitis C Study Group," Kidney International, vol. 56, pp. 700-706, 1999.

[50] T. Casanovas Taltavull, C. Baliellas Comellas, and J. M. Cruzado Garrit, "Results of hepatitis C virus treatment in patients on hemodialysis: data from published meta-analyses in 2008," Transplantation Proceedings, vol. 41, no. 6, pp. 2082 2084, 2009.

[51] S. C. Akhan, B. Kalender, and M. Ruzgar, "The response to pegylated interferon alpha $2 \mathrm{a}$ in haemodialysis patients with hepatitis C virus infection," Infection, vol. 36, no. 4, pp. 341344, 2008.

[52] D. N. Amarapurkar, N. D. Patel, and A. L. Kirpalani, "Monotherapy with peginterferon alpha-2b $12 \mathrm{kDa}$ for chronic hepatitis $\mathrm{C}$ infection in patients undergoing haemodialysis," Tropical gastroenterology, vol. 28, no. 1, pp. 16-18, 2007.

[53] H. W. Reesink, S. Zeuzem, C. J. Weegink et al., "Rapid decline of viral RNA in hepatitis C patients treated with VX-950: a phase Ib, placebo-controlled, randomized study," Gastroenterology, vol. 131, no. 4, pp. 997-1002, 2006.

[54] J. McHutchison and M. Sulkowski, "Scientific rationale and study design of the individualized dosing efficacy vs flat dosing to assess optimal pegylated interferon therapy (IDEAL) trial: determining optimal dosing in patients with genotype 1 chronic hepatitis C," Journal of Viral Hepatitis, vol. 15, no. 7, pp. 475-481, 2008.

[55] C. Hézode, N. Forestier, G. Dusheiko et al., "Telaprevir and peginterferon with or without ribavirin for chronic $\mathrm{HCV}$ infection," New England Journal of Medicine, vol. 360, no. 18, pp. 1839-1850, 2009.

[56] S. K. Gupta, S. L. Rosenkranz, Y. S. Cramer et al., "The pharmacokinetics and pharmacogenomics of efavirenz and lopinavir/ritonavir in HIV-infected persons requiring hemodialysis," AIDS, vol. 22, no. 15, pp. 1919-1927, 2008.

[57] H. Izzedine, V. Launay-Vacher, M. Legrand, D. Lieberherr, E. Caumes, and G. Deray, "ABT 378/r: a novel inhibitor of HIV-1 protease in haemodialysis," AIDS, vol. 15, no. 5, pp. 662-664, 2001.
[58] B. Domínguez-Gil, N. Esforzado, J. M. Campistol, A. Andres, and J. M. Morales, "Use of hepatitis C-positive donors for kidney transplantation," Transplantation Reviews, vol. 21, no. 4, pp. 195-203, 2007.

[59] L. M. Kucirka, A. L. Singer, R. L. Ros, R. A. Montgomery, N. N. Dagher, and D. L. Segev, "Underutilization of hepatitis Cpositive kidneys for hepatitis C-positive recipients," American Journal of Transplantation, vol. 10, no. 5, pp. 1238-1246, 2010.

[60] J. M. Morales, J. M. Campistol, B. Domínguez-Gil et al., "Long-term experience with kidney transplantation from hepatitis C-positive donors into hepatitis C-positive recipients," American Journal of Transplantation, vol. 10, no. 11, pp. 24532462, 2010.

[61] T. Schussler, C. Staffeld-Coit, J. Eason, and S. Nair, "Severe hepatitis $\mathrm{C}$ infection in a renal transplant recipient following hepatitis C genotype mismatch transplant," American Journal of Transplantation, vol. 4, no. 8, pp. 1375-1378, 2004.

[62] S. Ciesek, E. Steinmann, M. Iken et al., "Glucocorticosteroids increase cell entry by hepatitis C virus," Gastroenterology, vol. 138 , no. 5, pp. $1875-1884,2010$.

[63] J. Ortiz, J. Horton, A. Bingaman et al., "Campath 1-H induction and steroid-free maintenance immunosuppression $\mathrm{n}$ hepatitis C positive kidney recipients," Transplantation, vol. 86, no. 2S, p. 712, 2008.

[64] E. Akalin, B. Murphy, V. Sehgal, S. Ames, L. Daly, and J. S. Bromberg, "Rapid steroid withdrawal in hepatitis C viruspositive kidney transplant recipients," Clinical Transplantation, vol. 18, no. 4, pp. 384-389, 2004.

[65] P. A. Gallay, "Cyclophilin inhibitors," Clinics in Liver Disease, vol. 13, no. 3, pp. 403-417, 2009.

[66] F. U. L. Luan, D. E. Schaubel, H. Zhang et al., "Impact of immunosuppressive regimen on survival of kidney transplant recipients with hepatitis C," Transplantation, vol. 85, no. 11, pp. 1601-1606, 2008.

[67] N. Kamar, K. Sandres-Sauné, and L. Rostaing, "Influence of rituximab therapy on hepatitis $\mathrm{C}$ virus RNA concentration in kidney-transplant patients," American Journal of Transplantation, vol. 7, no. 10, p. 2440, 2007.

[68] L. Alric, V. DiMartino, J. Selves et al., "Long-term impact of renal transplantation on liver fibrosis during hepatitis $\mathrm{C}$ virus infection," Gastroenterology, vol. 123, no. 5, pp. 1494-1499, 2002.

[69] N. Kamar, L. Rostaing, J. Selves et al., "Natural history of hepatitis C virus-related liver fibrosis after renal transplantation," American Journal of Transplantation, vol. 5, no. 7, pp. 17041712, 2005.

[70] L. Castera, X. Forns, and A. Alberti, "Non-invasive evaluation of liver fibrosis using transient elastography," Journal of Hepatology, vol. 48, no. 5, pp. 835-847, 2008.

[71] B. Coco, F. Oliveri, A. M. Maina et al., "Transient elastography: a new surrogate marker of liver fibrosis influenced by major changes of transaminases," Journal of Viral Hepatitis, vol. 14, no. 5, pp. 360-369, 2007.

[72] R. Muñoz, E. Ramírez, I. Fernandez et al., "Correlation between fibroscan, liver biopsy, and clinical liver function in patients with hepatitis $\mathrm{C}$ virus infection after renal transplantation," Transplantation Proceedings, vol. 41, no. 6, pp. 24252426, 2009.

[73] D. Hestin, F. Guillemin, N. Castin, A. Le Faou, J. Champigneulles, and M. Kessler, "Pretransplant hepatitis $\mathrm{C}$ virus infection: a predictor of proteinuria after renal transplantation," Transplantation, vol. 65, no. 5, pp. 741-744, 1998. 
[74] J. M. Cruzado, S. Gil-Vernet, G. Ercilla et al., "hepatitis $\mathrm{C}$ virus-associated membranoproliferative glomerulonephritis in renal allografts," Journal of the American Society of Nephrology, vol. 7, no. 11, pp. 2469-2475, 1996.

[75] J. M. Morales, "hepatitis C virus infection and renal disease after renal transplantation," Transplantation Proceedings, vol. 36, no. 3, pp. 760-762, 2004.

[76] D. Roth, R. Cirocco, K. Zucker et al., "De novo membranoproliferative glomerulonephritis in hepatitis $\mathrm{C}$ virus- infected renal allograft recipients," Transplantation, vol. 59, no. 12, pp. 1676-1682, 1995.

[77] J. M. Morales, J. M. Campistol, A. Andrés, and J. L. Rodicio, "Glomerular diseases in patients with hepatitis C virus infection after renal transplantation," Current Opinion in Nephrology and Hypertension, vol. 6, no. 6, pp. 511-515, 1997.

[78] C. M. Meyers, L. B. Seeff, C. O. Stehman-Breen, and J. H. Hoofnagle, "hepatitis C and renal disease: an update," American Journal of Kidney Diseases, vol. 42, no. 4, pp. 631$657,2003$.

[79] N. Kamar, L. Rostaing, A. Boulestin et al., "Evolution of hepatitis $\mathrm{C}$ virus quasispecies in renal transplant patients with de novo glomerulonephritis," Journal of Medical Virology, vol. 69, no. 4, pp. 482-488, 2003.

[80] S. Baid, M. Pascual, W. W. Williams et al., "Renal thrombotic microangiopathy associated with anticardiolipin antibodies in hepatitis C-positive renal allograft recipients," Journal of the American Society of Nephrology, vol. 10, no. 1, pp. 146-153, 1999.

[81] F. G. Cosio, D. D. Sedmak, M. L. Henry et al., "The high prevalence of severe early posttransplant renal allograft pathology inhepatitis C positive recipients," Transplantation, vol. 62, no. 8, pp. 1054-1059, 1996.

[82] S. Baid, N. Tolkoff-Rubin, S. Saidman et al., "Acute humoral rejection in hepatitis $\mathrm{C}$-infected renal transplant recipients receiving antiviral therapy," American Journal of Transplantation, vol. 3, no. 1, pp. 74-78, 2003.

[83] N. Kamar, K. Sandres-Saune, J. Selves et al., "Long-term ribavirin therapy in hepatitis $\mathrm{C}$ virus-positive renal transplant patients: effects on renal function and liver histology," American Journal of Kidney Diseases, vol. 42, no. 1, pp. 184-192, 2003.

[84] A. A. Shahin, S. M. El Desouky, and H. S. Zayed, "A retrospective analysis of treatment outcomes in patients with hepatitis $\mathrm{C}$ related systemic vasculitis receiving intravenous methylprednisolone and cyclophosphamide," Clinical Rheumatology. In press.

[85] T. Namba, R. Shiba, T. Yamamoto et al., "Successful treatment of HCV-related cryoglobulinemic glomerulonephritis with double-filtration plasmapheresis and interferon combination therapy," Clinical and Experimental Nephrology, vol. 14, no. 4, pp. 372-376, 2010.

[86] F. Zaja, S. De Vita, C. Mazzaro et al., "Efficacy and safety of rituximab in type II mixed cryoglobulinemia," Blood, vol. 101, no. 10 , pp. 3827-3834, 2003.

[87] D. Roccatello, S. Baldovino, D. Rossi et al., "Long-term effects of anti-CD20 monoclonal antibody treatment of cryoglobulinaemic glomerulonephritis," Nephrology Dialysis Transplantation, vol. 19, no. 12, pp. 3054-3061, 2004.

[88] G. Basse, D. Ribes, N. Kamar et al., "Rituximab therapy for mixed cryoglobulinemia in seven renal transplant patients," Transplantation Proceedings, vol. 38, no. 7, pp. 2308-2310, 2006.
[89] M. Iyoda, K. L. Hudkins, S. Becker-Herman et al., "Imatinib suppresses cryoglobulinemia and secondary membranoproliferative glomerulonephritis," Journal of the American Society of Nephrology, vol. 20, no. 1, pp. 68-77, 2009.

[90] R. D. Bloom and J. R. Lake, "Emerging issues in hepatitis C virus-positive liver and kidney transplant recipients," American Journal of Transplantation, vol. 6, no. 10, pp. 2232-2237, 2006.

[91] R. D. Bloom, V. Rao, F. Weng, R. A. Grossman, D. Cohen, and K. C. Mange, "Association of hepatitis $\mathrm{C}$ with posttransplant diabetes in renal transplant patients on tacrolimus," Journal of the American Society of Nephrology, vol. 13, no. 5, pp. 13741380, 2002.

[92] B. L. Kasiske, J. J. Snyder, D. Gilbertson, and A. J. Matas, "Diabetes mellitus after kidney transplantation in the United States," American Journal of Transplantation, vol. 3, no. 2, pp. 178-185, 2003.

[93] S. Baid-Agrawal, U. Frei, P. Reinke et al., "Impaired insulin sensitivity as an underlying mechanism linking hepatitis $\mathrm{C}$ and posttransplant diabetes mellitus in kidney recipients," American Journal of Transplantation, vol. 9, no. 12, pp. 27772784, 2009.

[94] O. Heisel, R. Heisel, R. Balshaw, and P. Keown, "New onset diabetes mellitus in patients receiving calcineurin inhibitors: a systematic review and meta-analysis," American Journal of Transplantation, vol. 4, no. 4, pp. 583-595, 2004. 


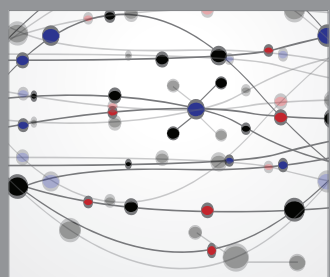

The Scientific World Journal
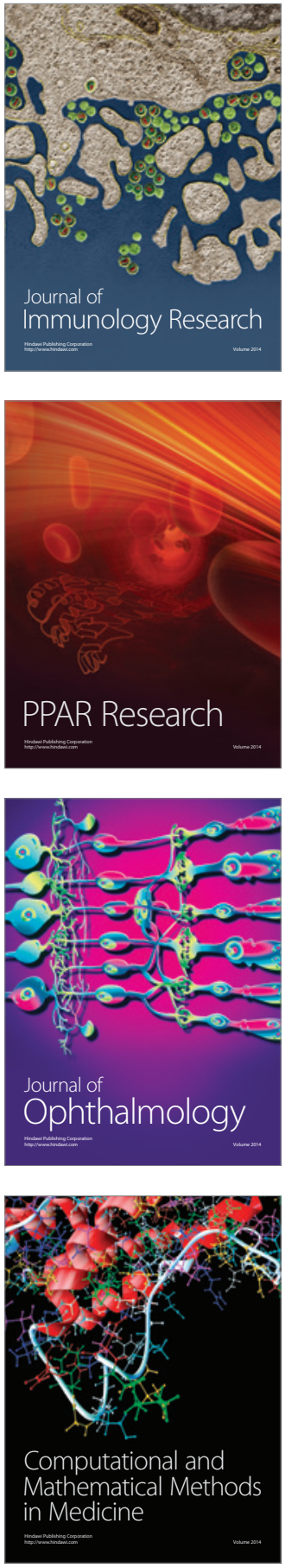

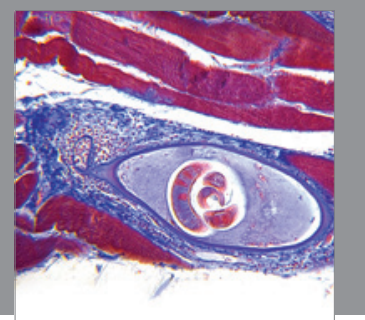

Gastroenterology

Research and Practice
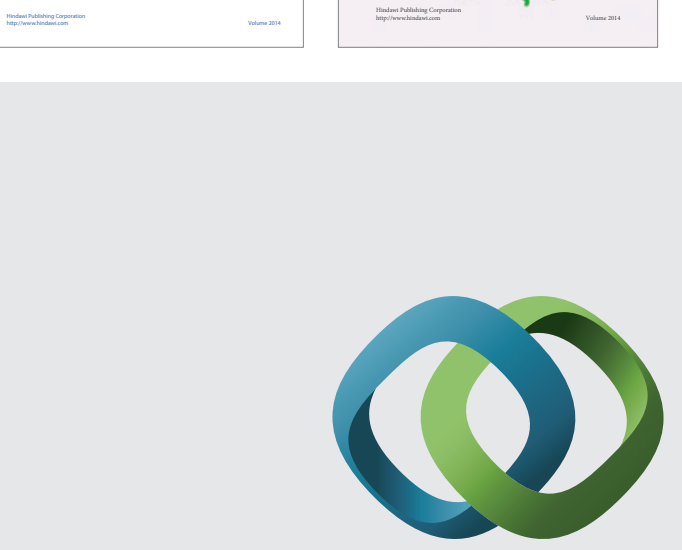

\section{Hindawi}

Submit your manuscripts at

http://www.hindawi.com
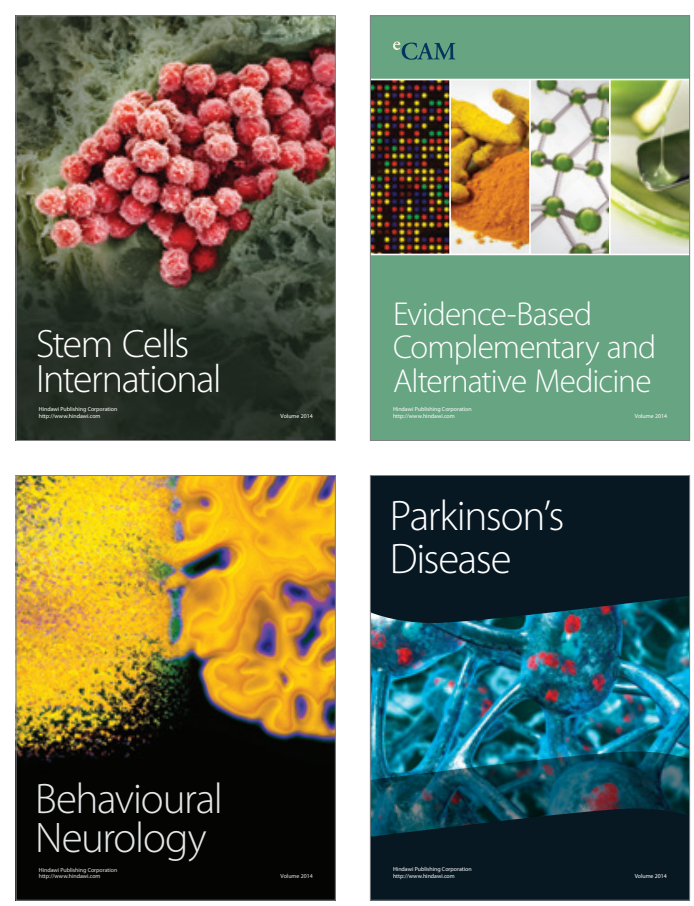

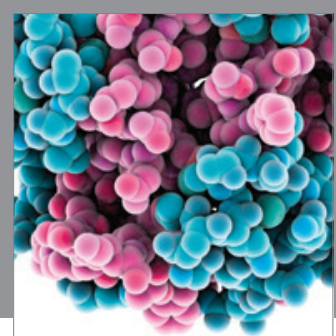

Journal of
Diabetes Research

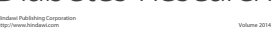

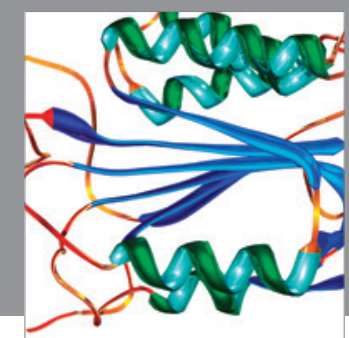

Disease Markers
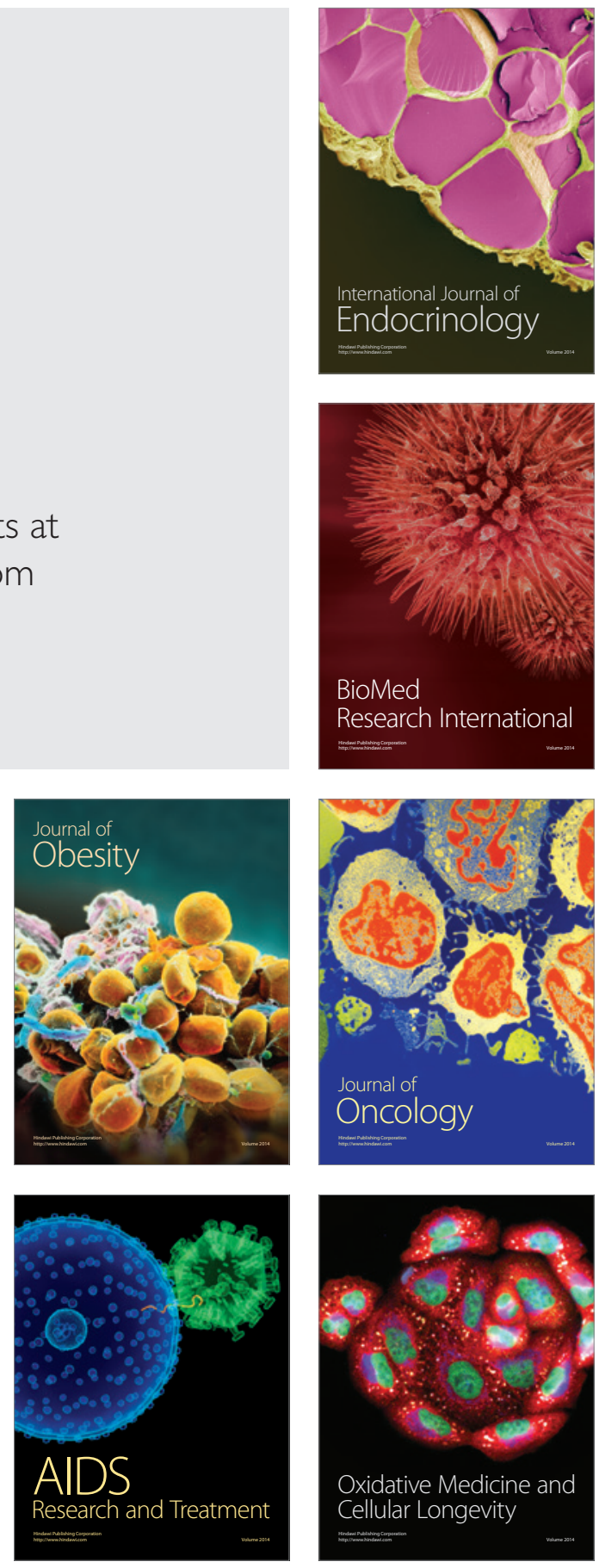